\title{
Burkitt's Lymphoma: Relationship of Infection and Case Clustering
}

\begin{abstract}
Malaria and Epstein-Barr virus (EBV) infection are recognized cofactors in the genesis of endemic Burkitt's lymphoma, although their relative contribution is not understood. Burkitt's lymphoma, the most common pediatric cancer in equatorial Africa, is a high-grade $\mathrm{B}$ cell lymphoma characterized by c-myc translocation. EBV is a ubiquitous B lymphotropic virus that persists in a latent state after primary infection, and in Africa, most children have sero-conversion by 3 years of age. Malaria infection profoundly affects the B cell compartment, inducing polyclonal activation and hyper-gammaglobulinemia. Specifically, acute malarial infection impairs the EBV-specific immune responses with the consequent increase in the number of EBVcarrying B cells in the circulation. ${ }^{1}$
\end{abstract}

The HIV epidemic has challenged our previous understanding of endemic Burkitt's lymphoma. Despite the strong association of Burkitt's lymphoma and HIV infection in the developed world, and against previous postulations that the cancer is due to immunosupression among African children, the HIV epidemic in the malaria belt has not been associated with a corresponding increase in incidence of childhood Burkitt's lymphoma. Even outside the context of HIV infection, there is substantial evidence for a strong but skewed immune response towards a Th2 response in genesis of Burkitt's lymphoma. Rather than a global and/or profound immunosupression, the final common pathway in genesis of Burkitt's lymphoma is the dysregulation of the immune response towards a Th2 response dominated by B-lymphocytes, and the concomitant suppression of the Th1 cellmediated immune surveillance, driven by various viral/parasitic/bacterial infections. ${ }^{2}$

Malaria and EBV are ubiquitous within the lymphoma belt of Africa, and, unless other cofactors are involved, the tumour should be much more common than it is. Malaria and EBV alone cannot account for the occasional 
shifting foci and space-time case clusters of endemic Burkitt lymphoma. Arboviruses and plant tumour promoters are other possible local cofactors that could explain such characteristics. The geographical and age distributions of endemic Burkitt's lymphoma parallel those of potentially oncogenic, mosquito-borne arboviruses. Arboviruses seem to be associated with case clusters of endemic Burkitt's lymphoma, and symptoms compatible with arbovirus infection have been seen immediately before the onset of the tumour. $^{3}$

In India, both EBV and malaria are very common. An interesting observation of clustering of cases of Burkitt's lymphoma has been made by the authors at their center. ${ }^{4}$ It would be interesting to study the relationship of Burkitt's lymphoma in our country with $\mathrm{EBV}$, malaria and other viral infections which may be able to explain this clustering of cases.

\section{REFERENCES:}

1. Moormann AM, Chelimo K, Sumba PO, Tisch DJ, Rochford R, Kazura JW. Exposure to holoendemic malaria results in suppression of Epstein-Barr virusspecific T cell immunosurveillance in Kenyan children. $J$ Infect Dis 2007;195:799-808.

2. Lubega J. T-helper 1 versus T-helper 2 lymphocyte immunodysregulation is the central factor in genesis of Burkitt lymphoma: hypothesis. Infect Agent Cancer 2007;2:10.

3. van den Bosch CA. Is endemic Burkitt's lymphoma an alliance between three infections and a tumour promoter? Lancet Oncol. 2004;5:738-46.

4. Bosco JIE, Appaji L, Aruna K, et.al. Clinical and radiological features of pediatric Burkitt's lymphoma - A four year study. Ind J. Med \& Paed Oncol. 2007;28(4):14-17.

Sameer Bakhshi Department of Medical Oncology All India Institute of Medical Sciences

New Delhi-29

Email:sambakh@hotmail.com 